\title{
Integrated Controls-Structures Design Methodology: Redesign of an Evolutionary Test Structure
}

\author{
Peiman G. Maghami \\ Sandeep Gupta \\ Kenny B. Elliot \\ Suresh M. Joshi \\ NASA Langley Research Center \\ Hampton, Virginia 23681
}

\begin{abstract}
An optimization-based integrated controls-structures design methodology for a class of flexible space structures is described, and the phase-0 Controls-Structures-Integration evolutionary model, a laboratory testbed at NASA Langley, is redesigned using this integrated design methodology. The integrated controls-structures design is posed as a nonlinear programming problem to minimize the control effort required to maintain a specified line-of-sight pointing performance, under persistent white noise disturbance. Static and dynamic dissipative control strategies are employed for feedback control, and parameters of these controllers are considered as the control design variables. Sizes of strut elements in various sections of the CEM are used as the structural design variables. Design guides for the struts are developed and employed in the integrated design process, to ensure that the redesigned structure can be effectively fabricated. The superiority of the integrated design methodology over the conventional design approach is demonstrated analytically by observing a significant reduction in the average control power needed to maintain specified pointing performance with the integrated design approach.
\end{abstract}




\section{Introduction}

Currently spacecraft are designed in separate iterative sequences within the structural and control disciplines. The structural design, performed first, takes into account loading considerations that would occur during launch, reboost, or operational maneuvers. The sizes and masses of mission-related components are estimated and a structure is designed which maintains the desired spatial relationships among various components during operations. In the next stage, a controller is designed for the fixed structure to orient, guide, and move the spacecraft to obtain the required spacecraft performance. Measures of spacecraft performance may take different forms such as limits on pointing jitter, transient response deviations, control power requirements, and so on. The control design must also provide satisfactory closed-loop stability robustness. This separate-discipline approach has been used successfully in most of the missions in the past. It works well when a relatively highstiffness structure is feasible, the nonstructural components are concentrated masses and inertias; and the performance requirements of the missions are not stringent. In these cases the structural modes are beyond the controlled bandwidth, so that there is minimal controlstructure interaction. However, this approach will not meet the stringent performance requirements of future space structures. A number of future space missions will utilize flexible structures in low-Earth orbits and geostationary orbits. Examples of such missions include space science platforms, space processing facilities, and Earth observation systems. Such missions typically require distributed-mass components such as booms, solar arrays, and antennas, whose dimensions range from a few meters to possibly hundreds of meters. To minimize the costs of construction, launching, and operations, it is necessary to make the structure as light as possible. However, the combination of large size and low structural 
mass leads to increased flexibility and makes attitude control of the structure to a specified precision more difficult.

Controls-structures interaction (CSI) in the form of destabilizing spillover ${ }^{1,2}$ has been verified in simple Earth-based laboratory experiments as well as in the design, analysis, ground development, test, and flight operation of space systems in industry ${ }^{1}$. The current approach to solving CSI problems is to design the spacecraft to avoid undesired dynamical interaction. This generally requires either stiffening the structure or slowing down the control system response. Stiffening the structure simplifies the control design problem, in that the predominant dynamics tend toward rigid body, but is costly in terms of mass as well as launch packaging, leading to increased fuel consumption. Slowing down the control response produces control inputs which have less of a chance of producing destabilizing effects, but is costly in terms of reduced performance capability. Neither approach is completely satisfactory. What is needed is a new design approach which has the capability of avoiding any damaging aspects of controls-structures interaction, while at the same time identifying and exploiting the beneficial aspects.

A number of studies have been presented in the past decade using integrated controlstructure design methodologies to enhance the overall performance of the controlled system. Minimization of structural mass, subject to constraints on closed-loop eigenvalues, damping parameters, and structural natural frequencies, has been considered in Refs. 3 and 4 . While dependence of control cost on the structural parameters is clear, since system matrices are functions of structural parameters, total structural mass is not considered directly as a function of control parameters, or control effort, in these studies. The effect of variable actuator masses, as a function of control effort, is addressed in Refs. 5 and 6. 
Other studies $^{4,7}$ have considered minimization of a control objective such as the norm of feedback gain matrices, as a measure of control effort, with constraints on closed-loop responses and total structural mass. A covariance approach is used in Ref. 8 to constrain overall system response with bounds on the allowable control energy. Multiobjective optimization has been used to optimize a combination of structural objectives such as structural mass, and control objectives, such as control energy or quadratic objective functions obtained from linear regulator theory ${ }^{9-11}$. Multiobjective optimization provides tradeoffs between competing objectives, thus providing a continuum of system designs, with different performance characteristics. Reference 12 includes a good survey of numerous other approaches employed for integrated control-structure design. However, most of the techniques in the literature have been applied to simple analytical models or laboratory apparatuses. Only recently have there been some studies in integrated design of large-order flexible space structures (with thousands of degrees of freedom) $5,6,13,14$.

The CSI program at NASA Langley ${ }^{15}$ has been developing an integrated controlsstructures design methodology which is described in this paper. The design methodology is based on the recognition that a high degree of coupling exists between the control and structural disciplines in the control of flexible space structures. Rather than performing separate structural and control designs, a unified environment for integrated controlstructural modelling, analysis and synthesis is developed. Within this environment, a design iteration consists of updating all critical (control and structure) design variables in a single integrated computational framework by assessing controlled performance with the current design variables. Parameter optimization techniques, employing nonlinear mathematical programming, are used for the synthesis of an optimal integrated structure. Realistic 
constraints, which have not been considered in previous literature, were imposed on the current design process, to ensure that the structure obtained through integrated design could be practically fabricated. For example, the strut designs were obtained using design curves relating effective areas and effective densities that imposed the requirements of manufacturability on the design process. This structure was subsequently used to verify the benefits of control/structure integrated design experimentally, as described in Ref. 16.

The phase-0 CSI evolutionary model (CEM), a laboratory test structure at NASA Langley (shown in Fig. 1), was used as the candidate flexible structure for controlsstructures integrated redesign. Two dissipative control strategies, namely, static dissipative and dynamic dissipative controllers, were employed for feedback compensation, since these compensators guarantee closed-loop stability in the presence of unmodelled dynamics and parametric uncertainties $^{17}$. The performance measure for optimization is the average control energy required to maintain prescribed line-of-sight pointing, in response to persistent, white noise at specified disturbance locations. Integrated redesign of the phase-0 CEM was performed with the two control strategies to improve the performance of the controlled system. Concurrently to the integrated design process, control-optimized dissipative controllers were designed for the nominal phase-0 CEM. A comparison of the controlled performance of the integrated designs with that of the conventional control-optimized designs indicate over $40 \%$ reduction in control power, while providing the same pointing performance. These results clearly demonstrate the advantage of integrated controlsstructures design methodology over the traditional sequential approach. The redesigned structure was fabricated and assembled in the laboratory and a number of active control experiments were performed with it to experimentally validate these results. This effort 
represents the first experimental validation of the integrated design methodology for realistic flexible space structures, and is described in Ref. 16.

\section{Mathematical Models}

\section{Structural Model}

The dynamics of flexible space structures can be treated as dynamics of a distributed parameter system from a continuum mechanics viewpoint. Applying the principles of classical mechanics to the distributed parameter system results in a system of partial differential equations with associated initial and boundary conditions. However, this framework for description of structural dynamics is of limited value in characterizing the dynamics of realistic, flexible space structures. Space structures are conventionally analyzed using approximate techniques such as the finite element method. A finite-dimensional, linear, time-invariant, mathematical model of a flexible space structure is given as

$$
M \ddot{x}+D \dot{x}+K x=\hat{B} u+\tilde{H} w
$$

where $x$ is an $n \times 1$ displacement vector; $u$ is the $m \times 1$ control input vector; $w$ is the $p \times 1$ disturbance vector; $M$ is the positive-definite inertia matrix; $D$ is the positive semidefinite open-loop damping matrix; $K$ is the positive semi-definite stiffness matrix; $\tilde{B}$ is an $n \times m$ control influence matrix; and $\hat{H}$ is $n \times p$ disturbance influence matrix. The rate and performance output equations are

$$
y_{r}=\tilde{C}_{r} \dot{x} ; \quad y_{p e r}=\tilde{C}_{p e r} x
$$

where $y_{r}$ is the rate measurement; $y_{p e r}$ is a performance vector; and $\tilde{C}_{r}$, and $\tilde{C}_{\text {per }}$ are the rate output and performance output influence matrices, respectively. The second-order 
representation of the structure given in Eq. (1) is obtained by finite element modeling of the structure, and the order of these models can be quite large. For design and analysis purposes, the order of the system is reduced using a modal truncation approach, wherein significant modes in the input-output characterization of the plant are retained and the remaining modes are truncated. The system equations in modal coordinates for the retained modes are written as

$$
\begin{aligned}
M_{r} \ddot{q}_{r}+D_{r} \dot{q}_{r}+K_{r} q_{r} & =\Phi^{T} \tilde{B} u+\Phi^{T} \tilde{H} w \\
& \equiv \Gamma_{c}^{T} u+\Gamma_{d}^{T} w
\end{aligned}
$$

where $q_{r}$ is an $r \times 1$ vector of modal amplitudes; $M_{r}, D_{r}, K_{r}$ are, respectively, the generalized inertia, damping and stiffness matrices; and $\Phi$ is an $n \times r$ matrix whose columns are the $r$ structural eigenvectors associated with the included modes. If the mode shapes are normalized with respect to the inertia matrix, and modal damping is assumed, then $M_{r}=I_{r \times r}, D_{r}=\operatorname{Diag}\left\{2 \zeta_{1} \omega_{1}, \ldots, 2 \zeta_{r} \omega_{r}\right\}$, and $K_{r}=\operatorname{Diag}\left\{\omega_{1}^{2}, \ldots, \omega_{r}^{2}\right\}$ where $\omega_{i}$ and $\zeta_{i}, i=1, \ldots, r$ are the open-loop frequencies and damping ratios. With collocated and compatible measurement sensors and control actuators,

$$
y_{r}=\tilde{B}^{T} \Phi \dot{q}_{r}=\Gamma_{c} \dot{q}_{r}
$$

Note that the collocation of these sensors and actuators is necessary for the implementation of the dissipative controllers. This collocation guarantees the system to be minimum phase, i.e, transmission zeros and poles of the system are in the left-half plane, and enhances the stability robustness of the overall system ${ }^{2}$.

Defining the state vector, $z=\left\{\begin{array}{ll}q_{r}^{T} & \dot{q}_{r}^{T}\end{array}\right\}^{T}$, the dynamics of the system can be written in a first-order form,

$$
\dot{z}=A z+B u+H w
$$


where

$$
A=\left[\begin{array}{cc}
0 & I_{r \times r} \\
-K_{r} & -D_{r}
\end{array}\right] ; \quad B=\left[\begin{array}{c}
0 \\
\Gamma_{c}^{T}
\end{array}\right] ; \quad H=\left[\begin{array}{c}
0 \\
\Gamma_{d}^{T}
\end{array}\right]
$$

The rate output and performance output vectors are given by

$$
y_{r}=\left[\begin{array}{ll}
0 & \Gamma_{c}
\end{array}\right] z \equiv C_{r} z ; \quad y_{p e r}=\left[\begin{array}{ll}
\tilde{C}_{p e r} \Phi & 0
\end{array}\right] z \equiv C_{p e r} z
$$

Here, $C_{p e r}$ is the output influence matrix associated with the performance output, $y_{p e r}$.

\section{Controller Design Methods}

Control system design for flexible space structures is a challenging problem because of their special dynamic characteristics, which include a large number of structural modes within the controller bandwidth; low, closely-spaced structural frequencies; very small inherent damping; and a lack of accurate knowledge of modal parameters. Moreover, the controller must be of a reasonably low order to be implementable; and must also satisfy the performance specifications (i.e., constraints on root-mean-square (RMS) pointing error, or the desired closed-loop bandwidth). The controller must also have robustness to nonparametric uncertainties, such as unmodeled structural modes, and to parametric uncertainties, that is, errors in the knowledge of the design model.

Two major categories of controller design methods for flexible space structures are model-based controllers and dissipative controllers. A model-based controller generally consists of a state estimator (a Kalman-Bucy filter, or some other observer) followed by a state feedback controller. The state estimator utilizes the knowledge of the design model in its prediction part. Using multivariable frequency-domain design methods, such controllers can be made robust to unmodeled structural dynamics, for example, the spillover effect 
can be overcome ${ }^{2}$. However, model-based controllers generally tend to be very sensitive to uncertainties in the design model, in particular, to uncertainty in the structural mode frequencies ${ }^{2,17}$. An analytical explanation of this instability mechanism may be found in Ref. 17.

Dissipative controllers utilize special passivity properties of structural dynamics, with collocated and compatible actuators and sensors, and provide robust stability in the presence of both nonparametric and parametric uncertainties ${ }^{2,17}$. Therefore, dissipative controllers have been used for feedback control in the integrated design methodology.

\section{A. Static Dissipative Controller}

The simplest dissipative controller is the static or constant-gain dissipative controller. Using collocated and compatible actuators and sensors, such as torquers with attitude and attitude rate sensors, and thrusters with linear position and velocity sensors, the constantgain dissipative control law is given by:

$$
u=-G_{r} y_{r}-G_{p} y_{p}
$$

where $y_{r}$ and $y_{p}$ are $m \times 1$ rate and position measurement vectors, where $m$ is the number of sensors; $G_{r}$ and $G_{p}$ are $m \times m$ symmetric, positive semi-definite rate and position gain matrices, respectively. This control law has been proven to give guaranteed closed-loop stability despite unmodeled elastic modes, parameter errors, certain types of actuator and sensor nonlinearities (such as saturation and dead zone), as long as actuator dynamics are limited to zero-order or first-order forms ${ }^{2}$. For space structures with zero frequency, rigidbody modes, position feedback is essential to ensure stability of the closed-loop system. 
However, for the ground-based structure considered in this paper, with non-zero frequency suspension modes, rate feedback alone is sufficient to guarantee stability. Therefore, for the integrated design and experimental studies, only rate feedback is considered. The drawback of this controller is that the achievable performance is inherently limited because of its simple mathematical structure.

\section{B. Dynamic Dissipative Controller}

To to obtain higher performance while still retaining the highly desirable robust stability, dynamic dissipative compensators can be used. An $n_{c^{-} \text {order, two-level, dynamic }}$ dissipative controller is given by:

$$
\begin{gathered}
\dot{x}_{c}=A_{c} x_{c}+B_{c} y_{r} \\
u=-C_{c} x_{c}-G_{r} y_{r}-G_{p} y_{p}
\end{gathered}
$$

where $A_{c}, B_{c}$, and $C_{c}$ are the compensator system, input, and output matrices, respectively. $G_{r}$ and $G_{p}$ are symmetric, positive semi-definite rate and position gain matrices corresponding to the static dissipative inner loop. For ground test articles, with no zero frequency rigidbody modes, position feedback is not necessary. The dynamic compensator, in the outer loop, is dissipative if its transfer function is strictly positive real. The positive realness lemma, or the Kalman-Yacubovich lemma ${ }^{18}$, assures this condition when $A_{c}$ is Hurwitz, $\left(A_{c}, B_{c}\right)$ is controllable, $\left(A_{c}, C_{c}\right)$ is observable and there exist a matrix $P=P^{T}>0$ such that

$$
\begin{gathered}
A_{c}^{T} P+P A_{c}=-Q \\
C_{c}=B_{c}^{T} P
\end{gathered}
$$


is satisfied, where $Q=Q^{T} \geq 0$. The main characteristic of dissipative controllers is that they do not rely on the knowledge of the design model to ensure stability. However, these controllers do use this knowledge to obtain the best possible performance. This controller assures robust asymptotic stability regardless of unmodeled structural dynamics or parametric uncertainties ${ }^{17}$.

\section{INTEGRATED DESIGN OF THE CEM}

This section presents the integrated design methodology, and its application to the redesign of the nominal phase-0 CEM.

The phase-0 CEM, shown in Fig. 1, is a laboratory testbed at NASA Langley, for experiments in control of flexible space structures. It consists of a 62-bay main truss (each bay is 10 inches long), two vertical towers, and two horizontal booms. The structure is suspended from the ceiling about 840 inches above the main truss using two cables attached to the horizontal booms, as shown in Fig. 1. A laser source is mounted at the top of the taller tower, referred to as the laser tower, and a reflector with a mirrored surface is mounted on the other tower, called the reflector tower. A laser beam is reflected by the mirrored surface onto a detector surface 660 inches above the reflector. The line-of-sight pointing problem is to maintain the laser close to its nominal position on the detector surface, despite persistent disturbances. Eight proportional bidirectional air thrusters, with maximum output force of $4.4 \mathrm{lbs}$ each, are available at stations 1 to 8 , shown in Fig. 1. Almost collocated with the thrusters at the eight stations are servo accelerometers to provide output measurements. Accelerometer signals are subsequently integrated with the aid of wash-out filters to provide the required rate information. A detailed description of the phase-0 CEM is available in 
Ref. 19.

The phase-0 CEM is representative of flexible space structures in that a number of low frequency closely spaced modes are within the bandwidth of its controllers, inherent damping is low, and its modal parameters are uncertain. However, one major difference between any ground-based structure, which must be suspended under the influence of gravity, and space structures is the absence of zero frequency rigid body modes in ground-based structures. The finite element model of the system has 3216 degrees of freedom; therefore, a large computational effort is required for solution of the structural eigenvalue problem of that size. The control design model consisted of the first 30 modes of the structure, which includes 24 flexible modes and six suspension modes (non-zero frequency, rigid-body modes, due to suspension of the structure in the presence of gravity). A modal damping ratio of $0.1 \%$ was assumed. The modal frequencies (in $\mathrm{Hz}$ ) of the first 10 modes of the nominal phase-0 CEM are $\{0.147,0.149,0.155,0.730,0.748,0.874,1.473,1.738,1.882,2.294\}$. The first six modes, ranging from $0.147 \mathrm{~Hz}$ to $0.874 \mathrm{~Hz}$, are the suspension modes. Modes 7 and 8 are the first two lateral and vertical bending modes, and mode 9 is the first torsional mode of this structure.

For the integrated design problem, white-noise disturbances of unit intensity are applied to the structure at stations 1 and 2, and the feedback control inputs are applied at stations 3 through 8. Past experience with the phase-0 CEM structure had shown that actuators at stations 7 and 8 easily destabilize the laser tower modes, in the form of spillover destabilization, which is typical in control of flexible structures. Therefore, even though actuators 7 and 8 are very efficient locations for exciting the structure, they were included for feedback control in order to consider the robust stability issue objectively. After 
actuators 7 and 8 , actuators 1 and 2 are the most effective actuators in exciting the structure. Therefore, actuators 1 and 2 were chosen as disturbance sources for integrated design and experimental validation. For the design process, no sensor and actuator dynamics were included. Thus, the system dynamics are given by Eq. (5), where the matrix $\Gamma_{c}$ contains the modal displacements at the control stations 3 through 8 ; and matrix $\Gamma_{d}$ contains modal displacements at the disturbance stations 1 and 2. The performance vector, $y_{p e r}$, is the lineof-sight pointing error or deviation of the laser point on the detector system from its nominal position in local $x$ and $y$ coordinates. The performance output matrix, $\tilde{C}_{p e r}$, is computed from the kinematics of small angular motion about the nominal configuration of the system.

The feedback control configuration used for the synthesis of the integrated controlsstructures design as well as the control-optimized design is as follows. Persistent disturbance noise, $w(t)$, is applied to the structure at the disturbance locations (stations 1 and 2 ), and the deviation of the line-of-sight pointing error vector, $y_{\text {per }}(t)$, as measured by the laser detector, is to be maintained within desired specifications. The feedback control system applies control inputs, $u(t)$, at control stations $3-8$, using the measured outputs, $y_{r}(t)$, at nearly collocated nodes.

The design optimization problem is to minimize the steady-state average control power while maintaining a specified root-mean-square (RMS) line-of-sight pointing error, and without exceeding the total mass budget of the nominal phase-0 CEM. Mathematically, the design problem is to minimize

$$
J=\lim _{t \rightarrow \infty}\left[\operatorname{Tr}\left(E\left\{u(t) u^{T}(t)\right\}\right)\right]
$$


with respect to structural and control design variables, subject to the constraints:

$$
\lim _{t \rightarrow \infty}\left[\operatorname{Tr}\left(E\left\{y_{p e r}(t) y_{p e r}^{T}(t)\right\}\right)\right]^{\frac{1}{2}} \leq y_{p e r}^{\max } ; \quad M_{s t r} \leq M_{\max }
$$

where $y_{p e r}^{\max }$ is the maximum allowable line-of-sight pointing error, $M_{\max }$ is the mass budget, $\operatorname{Tr}$ denotes trace of the matrix, and $E$ is the expectation operator.

For the redesign of the CEM, the limit on the root-mean-square line-of-sight pointing was chosen as $y_{\text {per }}^{\max }$ equal to $2.4 \mathrm{in}$, which is an order of magnitude reduction from the openloop RMS line-of-sight pointing for phase-0 CEM structure (which is $22.54 \mathrm{in}$ ); and $M_{\max }$ was chosen to be 1.92 lb.sec ${ }^{2} /$ in, which is the nominal mass of phase-0 CEM structure.

Using the static or dynamic dissipative controller, the closed-loop system dynamics can be written as

$$
\dot{\bar{x}}=\bar{A} \bar{x}+\bar{H} w ; \quad y_{p e r}=\bar{C}_{p e r} \bar{x} ; \quad u=\bar{C}_{u} \bar{x}
$$

where $\bar{x}$ is the state vector for the closed-loop dynamics, $w(t)$ is zero mean, white noise disturbance applied at the disturbance locations, $y_{p e r}$ is the line-of-sight pointing error, $u(t)$ is the control vector, and $\bar{A}, \bar{H}, \bar{C}_{\text {per }}$ and $\bar{C}_{u}$ are the corresponding closed-loop system matrices. The steady state covariance matrix for the closed loop state, $\Sigma_{\bar{x}}$, is computed by solving the following Lyapunov equation ${ }^{20}$

$$
\bar{A} \Sigma_{\bar{x}}+\Sigma_{\bar{x}} \bar{A}^{T}+\bar{H} \Sigma_{w} \bar{H}^{T}=0
$$

where $\Sigma_{w}$ is the covariance matrix for the disturbance noise, $w(t)$. The steady-state average control power is given as

$$
\lim _{t \rightarrow \infty} \operatorname{Tr}\left\{E\left\{u(t) u^{T}(t)\right\}\right\}=\operatorname{Tr}\left[\bar{C}_{u} \Sigma_{\bar{x}} \bar{C}_{u}^{T}\right]
$$


and root-mean-square line-of-sight pointing error is

$$
\lim _{t \rightarrow \infty}\left[\operatorname{Tr}\left(E\left\{y_{p e r}(t) y_{p e r}^{T}(t)\right\}\right)\right]^{\frac{1}{2}}=\left[\operatorname{Tr}\left(\bar{C}_{p e r} \Sigma_{\bar{x}} \bar{C}_{p e r}^{T}\right)\right]^{\frac{1}{2}}
$$

The total mass of the structure, $M_{s t r}$, is obtained from the mass matrix of the finite element analysis routines.

For the structural design, the CEM structure was divided into seven sections, shown in Fig. 2, namely, three sections in the main truss, one section for the laser tower, one section for the reflector tower, and one section for the two horizontal booms. The primary dynamic characteristics of these sections were defined by the mass and stiffness properties of the struts and node balls comprising these sections. A typical strut and node ball of the resigned CEM structure is shown in Fig. 3. The strut/node ball system was modeled as an axial rod between node ball centers representing the stiffness of the strut and node ball with a point mass at each end representing the mass of the node ball. The stiffness of the axial rods in each section were used as the structural design variables. From a modeling point of view the rod stiffness is defined by its effective area, length, and modulus. Given that the length and modulus of the struts were held constant, the effective area of the axial rod was used to quantify the stiffness of the struts. Therefore, three structural design variables were used in each section, namely, the effective areas of the longerons, battens and diagonals. Thus, the integrated design of the structure involved a total of 21 structural design variables.

In an ideal design with uniform struts, the effective area of the rod would be the crosssectional area of the strut. However, in this case the strut is a complex member with joints and variable cross-sections. What is required is a means to relate the effective area used to model the strut/node ball system to a strut that can be manufactured. The strut of Fig. 3 
is composed of three sections, namely, the node ball, tube, and connection hardware. The system is designed such that the node ball and connection hardware remain the same for all struts. However the tube portion of the strut can be changed. From a stiffness point of view the strut/node ball system can be thought of as three springs in series. The end springs represent the stiffness of half a node ball and connection hardware, while the center spring is the stiffness of the tube. The effective area can be represented as:

$$
A_{e f f}=\frac{\left(k_{c} k_{t}\right) \ell}{\left(2 k_{t}+k_{c}\right) E}
$$

where $k_{c}$ is the stiffness of the end springs, $k_{t}$ is the stiffness of the tube, $\ell$ is the distance between node ball centers, and $E$ is the modulus of elasticity. If the center-to-center length, modulus, and stiffness of the node ball and connection hardware remain constant, then the effective area will be governed by the stiffness of the center tube. Associated with each effective area is an effective density defined as

$$
\rho_{e f f}=\frac{m}{A_{e f f} \ell}
$$

where $m$ is the total mass of the strut, excluding the node ball mass. The effective density combined with the node ball mass is used to define the mass of the strut/ node ball system in the finite element analysis of the structure.

Now the stiffness and mass properties of the structure are functions of the geometry of the center tube of the strut. To ensure that the strut/node ball system could be manufactured, design guides were semi-empirically developed that defined a two dimensional design space of manufacturable struts ${ }^{21}$. The design space gives the relationship between the effective area and effective density for the strut design shown in Fig. 3. The strut design 
guides are shown in Fig. 4, where the dotted line corresponds to longerons and battons, and the solid line is the design guide for diagonals. The curve represents the minimum mass (density) strut for a given strut stiffness (effective area). The curve is continuous due to the manufacturing process. The center tube is manufactured by turning a stock tube to a specified outer diameter, thereby, allowing a variety of center tube stiffness. The design space is limited at low effective areas (towards the left end of the design guides in Fig. 4) by the strength of the strut, whereas the high effective area (towards the right end in Fig. 4 ) is limited by the linearity of the connection hardware. The control design variables for static and dynamic dissipative controllers are described in the next section.

An integrated design software tool, called CSI-DESIGN, being developed at the NASA Langley, was used to perform the parameter optimizations numerically. CSI-DESIGN uses an in-core database architecture and is composed of public domain software ${ }^{22-24}$. The package has control, structural and optimization modules linked together in a unified environment to perform design iterations on both structural and control design variables. A description of the contents of the CSI-DESIGN structural module may be found in Ref. 25. Employing a four-processor Alliant FX-80 digital computer, integrated optimization was performed using the Automated Design Synthesis (ADS) software ${ }^{22}$. This design optimization took about 8 hours of cpu time for each run. Gradient computations were performed using finite difference approximations. An interior penalty function method of ADS was used to solve the nonlinear programming problems. In this method, the constrained optimization problem is transformed into an unconstrained problem through creation of a pseudo-objective function, which is the sum of the original objective function and an imposed penalty function (a function of the constraints ${ }^{26}$ ). The Reverse-Cuthill- 
McKee algorithm ${ }^{27}$ for minimizing the bandwidth of the banded stiffness and mass matrices was used to reduce computational and memory requirements. Additionally, analytical expressions for eigenvalue/eigenvector sensitivity, with respect to the structural design variables ${ }^{28}$, were used in the integrated design process to approximate the eigenvalues and eigenvectors at design points which are in the neighborhood of the nominal design point. This approximation was in the form of a first-order Taylor series approximation and resulted in substantial computational savings since it removes the need for costly computation of structural eigenvalues and eigenvectors at many of the optimization moves. The Lyapunov equation for closed-loop state covariance matrix, Eq. (14), is solved by transforming the matrices to a Schur form and solving the resulting linear equations, as described in Refs. 23 and 29. The control design variables used for static and dynamic dissipative control, and the results of the integrated design process are described next.

\section{Integrated Design Results}

\section{A. Integrated Design with Static Dissipative Controller}

This section describes the control design variables for static dissipative controllers and the design optimization results. Since the test article does not have any zero frequency rigid body modes, only rate feedback is employed for the static dissipative controller, i.e.

$$
u=-G_{r} y_{r}
$$

where the rate gain matrix, $G_{r}$, is chosen to be a $6 \times 6$ diagonal matrix, whose elements constitute six control design variables. Note that a diagonal gain matrix is used for simplicity, and because the resulting decentralized controller generally exhibits superior 
performance robustness. The closed-loop matrices in Eq. (13) are

$$
\bar{A}=\left[\begin{array}{cc}
0 & I_{r \times r} \\
-K_{r} & -D_{r}-\Gamma_{c}^{T} G_{r} \Gamma_{c}
\end{array}\right] ; \quad \bar{H}=H ; \bar{C}_{p e r}=C_{p e r} ; \quad \bar{C}_{u}=-\left[0 G_{r} \Gamma_{c}\right] .
$$

Average control power and root-mean-square line-of-sight pointing error for static dissipative controllers, are computed using Eqs. (15)-(16). A total of 27 design variables, 21 structural design variables and 6 control design variables, were used for the integrated design optimization for the static dissipative integrated design.

The results of the design optimizations are summarized in Table 1. First, the average control power was minimized with respect to the control design variables, while the structural design variables are fixed at the nominal phase-0 values, namely, effective areas for battons and longerons being $0.134 \mathrm{in}^{2}$ and effective area for diagonals being $0.124 \mathrm{in}^{2}$. This corresponds to the conventional approach of synthesizing the best achievable controllers for a fixed structure. The control-optimized design required an average steady-state control power of $7.11 l b^{2}$ to maintain RMS line-of-sight error within the specified limit, that is, 2.4 in. Next, an integrated design was performed to minimize the average control power with respect to both control and structural design variables. The results (see Table 1) indicate an average control power of $4.21 l b^{2}$ to maintain the same RMS line-of-sight error. The integrated design results in a reduction of more than $40 \%$ in the average control power over the conventional design, for the same RMS line-of-sight pointing performance. The effective areas for the structural design are shown in Table 2. Comparing the new design variables with the effective areas of the nominal phase-0 CEM, it is observed that the longerons for all three sections of the main truss, particularly the section closest to the disturbance sources, are considerably stiffened. The longerons in the laser tower are also stiffened. On 
the contrary, the horizontal booms and the reflector tower became more flexible, partially to satisfy the mass constraint. Generally, all the diagonals and the battens decreased in size, to satisfy a constraint on the total mass i.e. the mass of the redesigned structure had to be less than or equal to the mass of the nominal phase-0 design. Consequently, mass was taken from the battens and diagonals and was redistributed to the longerons of some sections because longerons are quite effective in increasing the stiffness of that section. This trend may be attributed to a trade off between structural controllability and observability at the actuator stations, structural observability of the performance vector and structural excitability at the disturbance locations. The areas near the disturbance sources, i.e. stations 1 and 2, were stiffened in order to reduce the sensitivity of the structure to external disturbances at those locations, while ensuring that no appreciable loss of controllability and/or observability occurred at the control stations. The control gains for the control-optimized and the integrated designs are shown in Table 3 . Generally it is observed that the gains for the redesigned system are considerably lower than those for phase-0 CEM design (except for actuator 6). This is to be expected since the required control power for redesigned system is significantly lower than that of the nominal phase-0 CEM.

\section{B. Integrated Design with Dynamic Dissipative Controller}

The dynamic dissipative controller represented by Eqns. (7) and (8), with no static inner loop, that is, $G_{p}=0$ and $G_{r}=0$, was used for the integrated redesign of the structure. The compensator state matrix $A_{c}$ and compensator input influence matrix $B_{c}$, 
were assumed to be block diagonal, as shown below. where $A_{c i}$ and $B_{c i}, i=1,2, \ldots, 6$ are, respectively, $2 \times 2$ matrices and $2 \times 1$ vectors, defined as

$$
A_{c i}=\left[\begin{array}{cc}
0 & 1 \\
-\alpha_{i} & -\beta_{i}
\end{array}\right] \quad ; \quad B_{c i}=\left[\begin{array}{l}
0 \\
1
\end{array}\right]
$$

The parameters, $\alpha_{i}$ and $\beta_{i}$, for $i=1,2, \ldots, 6$, are the control design variables. Furthermore, the weighting matrix $Q$ in Eq. (9) was assumed to be diagonal, that is,

$$
Q=\operatorname{Diag}\left(q_{1}, q_{2}, \ldots, q_{12}\right)
$$

with $q_{j}$, for $j=1,2, \ldots, 12$, also being the control design variables. For the dynamic dissipative controller, the closed-loop system matrices in Eq. (13) are

$$
\bar{A}=\left[\begin{array}{cc}
A & -B C_{c} \\
B_{c} C_{r} & A_{c}
\end{array}\right] ; \quad \bar{H}=\left[\begin{array}{c}
H \\
0
\end{array}\right] ; \quad \bar{C}=\left[\begin{array}{ll}
C_{p e r} & 0
\end{array}\right]
$$

For average control power computation, $\bar{C}_{u}=-\left[\begin{array}{ll}0 & C_{c}\end{array}\right]^{T}$. Again, Eqns. (15)-(16) are used for computing control power and root-mean-square line-of-sight pointing error. Thus, the scalar variables $\alpha_{i}, \beta_{i}, i=1,2, \ldots, 6$ and $q_{j}, j=1,2, \ldots, 12$ constitute 24 control design variables. Alongwith 21 structural design variables, the total number of design variables for integrated redesign with dynamic dissipative controllers is forty five.

Table 1 shows the results of designs with the dynamic dissipative controller. The control-optimized design for phase-0 CEM required a control power of $6.41 \mathrm{lb}^{2}$ to maintain RMS line-of-sight pointing within $2.4 \mathrm{in}$. The integrated redesign reduces the average control power by $44 \%$ over the conventional design to $3.64 l b^{2}$. The effective areas for the structural elements for integrated design with the dynamic dissipative compensator are shown in Table 2. Effective areas for longerons, battens and diagonals for the integrated 
design with dynamic dissipative controllers show the same trends as that for the static dissipative controller. Control design variables for the control-optimized design variables and the integrated design are shown in Table 4 . Figure 5 compares the maximum and minimum singular value plots of op timal dynamic dissipative controllers for phase-0 CEM (in dashed lines) and redesigned system (in solid lines). It is observed that the controller gains are generally smaller throughout the frequency spectrum except in the very low frequency region.

The results obtained for both the static and dynamic dissipative controllers clearly show that integrated controls-structures design methodology can yield a substantially superior overall design over the conventional sequential design scenario. Moreover, Table 2 shows that the optimal structure for both dissipative control laws exhibit similar trends. In fact, the structural design variables for the two optimal structures are within $20 \%$ of each other. A structural design close to both these designs was chosen for fabrication and assembly in the laboratory. This redesigned structure was referred to as phase-1 CEM. A number of active control experiments were performed with both the structures, that is, phase-0 CEM and phase-1 CEM, to validate the benefits of integrated controls-structures design demonstrated analytically in this paper. This experimental validation is described in Ref. 16.

\section{Concluding Remarks}

An optimization-based integrated controls-structures design methodology has been presented in this paper, using static and dynamic dissipative compensators. To demonstrate the benefits of this design approach, the nominal phase-0 CEM structure was redesigned, using the integrated design methodology, to minimize the average control power required 
to maintain specified root-mean-square line-of-sight pointing error under persistent disturbances. The redesign with static dissipative compensators resulted in a $40 \%$ reduction in control power, whereas the redesign with dynamic dissipative compensators produced a $44 \%$ reduction in control power. Therefore, it has been demonstrated, analytically, that integrated controls-structures design can yield designs which are substantially superior to those obtained through the traditional sequential approach. Moreover, in order to show that the benefits achieved analytically can be realized in practice, the redesigned structure was fabricated and assembled in the laboratory. Experimental validation of these results has been performed successfully, and will be presented in a subsequent paper. Finally, this work demonstrates the capability of a software design tool (CSI-DESIGN) which implements the automated design procedure in a unified environment for structural and control designs.

\section{References}

1. Ketner, G. L., "Survey of Historical Incidences With Controls-Structures Interaction and Recommended Technology Improvements Needed to Put Hardware in Space," Battelle, Pacific Northwest Laboratory, Report No. PNL-6846, Richland, WA, March 1989.

2. Joshi, S. M., Control of Large Flexible Space Structures, edited by M. Thoma and A. Wyner, Volume 131, Lecture Notes in Control and Information Sciences, Springer-Verlag, Berlin, 1989.

3. Khot, N. S., Eastep, F. E., and Venkayya, V. B., "Simultaneous Optimal Structural/ Control Modifications to Enhance the Vibration Control of a Large Flexible Structure," AIAA Guidance, Navigation, and Control Conference, Snowmass, CO, August 1985, pp.1642-1647.

4. Khot, N. S., "An Integrated Approach to the Minimum Weight and Optimum Control Design of Space Structures," Large Space Structures: Dynamics and Control, edited by S. N. Atluri and A. K. Amos, Springer-Verlag, Berlin Heidelberg, 1988, pp. 355-363.

5. Woodard, S. E., Padula, S. L., Graves, P. C., and James, B. B., "An Optimization Method for Controlled Space Structures with Variable Actuator Mass," Fourth NASA/DoD Controls/ Structures Interaction Technology Conference, Orlando, FL, January 1990, pp. 411-428. 
6. Padula, S. L., James, B. B., Graves, P. C., and Woodard, S. E., "Multidisciplinary Optimization of Controlled Space Structures With Global Sensitivity Equations," NASA Langley Research Center, TP-3130, Hampton, VA, November 1991.

7. Bodden, D. S., and Junkins, J. L., "Eigenvalue Optimization Algorithms for Structure/Controller Design Iterations," AIAA Journal of Guidance, Control, and Dynamics, Vol. 8, No. 6, November-December 1985, pp. 697-706.

8. McLaren, M. D., and Slater, G. L., "A Covariance Approach to Integrated Control/ Structure Optimization," AIAA Dynamics Specialists Conference, Long Beach, CA, April 1990, pp. 189-205.

9. Junkins, J. L., and Rew, D. W., "A Simultaneous Structure/Controller Design Iteration Method," American Control Conference, Boston MA, June 1985, pp. 1642-1647.

10. Milman, M., Salama, M., Scheid, R., Bruno, R., and Gibson, J. S., "Integrated ControlStructure Design: A Multiobjective Approach,” Jet Propulsion Laboratory, JPL D-6767, Pasadena, CA, January 1990.

11. Gilbert, M. G., and Schmidt, D. K., "Integrated Structure/Control Law Design by Multilevel Optimization," AIAA Journal of Guidance, Control, and Dynamics, Vol. 14, No. 5, September-October 1991, pp. 1001-1007.

12. Rao, S. S., and Pan, T. S., "Robustness Improvement of Actively Controlled Structures Through Integrated Structural/Control Design," Flgiht Dynamics Directorate, Wright Laboratory, Report No. WL-TR-91-3058, Wright-Patterson Air Force Base, OH, May 1991.

13. Maghami, P. G., Joshi, S. M., Armstrong, E. S., and Walz, J. E., "Integrated ControlsStructures Design Methodology Development for a Class of Flexible Spacecraft," Third Air Force/NASA Symposium on Recent Advances in Multi-disciplinary Analysis and Optimization, San Fransisco, CA, September 1990, pp. 1-6.

14. Maghami, P. G., Joshi, S. M., and Armstrong, E. S., "An Optimization-Based Integrated Controls-Structures Design Methodology for Flexible Space Structures," NASA Langley Research Center, TP 3283, Hampton, VA, January 1993.

15. Newsom, J. R., Layman, W. E., Waites, H. B., and Hayduk, R. J., "The NASA ControlsStructures Interaction Technology Program," NASA Langley Research Center, TM102752, Hampton, VA, October 1990.

16. Maghami, P. G., Gupta, S., Elliot, K. B., and Walz, J. E., "Experimental Validation of an Integrated Controls-Structures Design Methodology," Journal of guidance, Control, and Dynamics, , Vol. 19, No.2, 1996,pp. 324-333. 
17. Joshi, S. M., and Maghami, P. G., "Dissipative Compensators for Flexible Spacecraft Control", IEEE Transactions on Aerospace and Electronic Systems, Vol. 28, No. 3, July 1992, pp. 768-774.

18. Lefschetz, S., Stability of Nonlinear Systems, Academic Press, New York, NY, 1965.

19. Belvin, W. K., Elliot, K. B., Horta, L. G., Bailey, J. P., Bruner, A. M., Sulla, J. L, Won, J., and Ugoletti, R. M., "Langley's CSI Evolutionary Model: Phase 0," NASA Langley Research Center, TM 104165, Hampton, VA, November 1991.

20. Stengel, R. F., Stochastic Optimal Control - Theory and Applications, John Wiley \& Sons, Inc., New York, NY, 1986.

21. Gronet, M. J., et al., "Design, Analysis, and Testing of the Phase 1 CSI Evolutionary Model Erectable Truss", NASA CR-4461, NASA Langley Research Center, Hampton, VA, August 1992.

22. Vanderplaats, G. N., "ADS - A Fortran Program for Automated Design Synthesis - Version 1.10", University of California, Santa Barbara, NASA CR-177985, Santa Barbara, CA, 1985.

23. Armstrong, E. S., "ORACLS - A System for Linear-Quadratic-Gaussian Control Law Design," NASA Langley Research Center, TP 1106, Hampton, VA, 1978.

24. Dongarra, J. J., Moler, C. B., Bunch, J. R., and Stewart, G. W., "Linpack User's Guide," Society for Industrial and Applied Mathematics (SIAM), Philadelphia, PA, 1979.

25. Belvin, W. K., and Park, K. C., "Computer Implementation of Analysis and Optimization Procedures for Control-Structure Interaction Problems," AIAA Dynamics Specialist Conference, Long Beach, CA, April 1990, pp. 32-41.

26. Vanderplaats, G. N., Numerical Optimization Techniques for Engineering Design: With Applications, McGraw-Hill, Inc., New York, NY, 1984.

27. George, A., and Liu, J. W.-H., Computer Solution of Large Sparse Positive-Definite Systems, Prentice Hall, Inc., Englewood Cliffs, NJ, 1984.

28. Kenny, S. P., Hou, G. J., and Belvin, W. K., "Eigensensitivity in Integrated Design", Fourth NASA/DoD Controls/Structures Interaction Technology Conference, Orlando, FL, January 1991, pp. 820-826.

29. Bartels, R. H., and Stewart, G. W., "Algorithm 432 - Solution of the Matrix Equation $A X+X B=C$," Communications of the ACM, Vol. 15, No. 9, September 1972. 
Table 1 Results of design study with static and dynamic dissipative controllers.

\begin{tabular}{lcc}
\hline \hline & $\begin{array}{c}\text { RMS Line-of-sight } \\
\text { Error (in) }\end{array}$ & $\begin{array}{c}\text { Control Power } \\
\left(\mathrm{lb}^{\wedge} 2\right)\end{array}$ \\
\hline $\begin{array}{l}\text { Control-Optimized Design with } \\
\text { Static Dissipative Controller }\end{array}$ & 2.4 & 7.11 \\
$\begin{array}{l}\text { Integrated Design with } \\
\text { Static Dissipative Controller }\end{array}$ & 2.4 & 4.21 \\
$\begin{array}{l}\text { Control-Optimized Design with } \\
\text { Dynamic Dissipative Controller }\end{array}$ & 2.4 & 6.41 \\
$\begin{array}{l}\text { Integrated Design with } \\
\text { Dynamic Dissipative Controller }\end{array}$ & 2.4 & 3.64 \\
\hline
\end{tabular}


Table 2 Structural design variables for integrated design with static and dynamic dissipative controllers.

\begin{tabular}{|c|c|c|c|c|c|c|c|c|c|c|}
\hline \multirow[b]{2}{*}{$\begin{array}{l}\text { Var. } \\
\text { No. }\end{array}$} & \multicolumn{2}{|c|}{ Longerons, in^2 } & \multicolumn{3}{|c|}{ Battens, in^2 } & \multicolumn{3}{|c|}{ Diagonals, in^2 } & \multirow[b]{2}{*}{$\begin{array}{l}\text { Sec. } \\
\text { No. }\end{array}$} & \multirow[b]{2}{*}{ Description } \\
\hline & Static & Dynamic & $\begin{array}{l}\text { Var. } \\
\text { No. }\end{array}$ & Static & Dynamic & $\begin{array}{l}\text { Var. } \\
\text { No. }\end{array}$ & Static & Dynamic & & \\
\hline 1 & 0.330 & 0.330 & 2 & 0.082 & 0.077 & 3 & 0.082 & 0.098 & 1 & main truss \\
\hline 4 & 0.085 & 0.080 & 5 & 0.083 & 0.087 & 6 & 0.085 & 0.087 & 2 & $\begin{array}{l}\text { horizontal } \\
\text { boom }\end{array}$ \\
\hline 7 & 0.173 & 0.142 & 8 & 0.082 & 0.086 & 9 & 0.082 & 0.082 & 3 & laser tower \\
\hline 10 & 0.260 & 0.295 & 11 & 0.082 & 0.080 & 12 & 0.081 & 0.066 & 4 & main truss \\
\hline 13 & 0.257 & 0.258 & 14 & 0.081 & 0.078 & 15 & 0.079 & 0.066 & 5 & main truss \\
\hline 16 & 0.095 & 0.100 & 17 & 0.081 & 0.077 & 18 & 0.079 & 0.066 & 6 & $\begin{array}{l}\text { horizontal } \\
\text { boom }\end{array}$ \\
\hline 19 & 0.096 & 0.117 & 20 & 0.081 & 0.083 & 21 & 0.082 & 0.083 & 7 & $\begin{array}{l}\text { reflector } \\
\text { tower }\end{array}$ \\
\hline
\end{tabular}


Table 3 Control design variables for integrated design, with static dissipative controllers.

\begin{tabular}{cccc}
\hline \hline $\begin{array}{c}\text { Var. } \\
\text { No. }\end{array}$ & $\begin{array}{c}\text { Control-Optimized } \\
\text { Design }\end{array}$ & $\begin{array}{c}\text { Integrated } \\
\text { Design }\end{array}$ & $\begin{array}{c}\text { Actuator } \\
\text { No. }\end{array}$ \\
\hline 1 & 1.2110 & 0.5586 & 3 \\
2 & 2.0634 & 0.6837 & 4 \\
3 & 1.2985 & 0.7899 & 5 \\
4 & 0.5352 & 1.1117 & 6 \\
5 & 1.5050 & 1.0201 & 7 \\
6 & 0.4593 & 0.5982 & 8 \\
\hline
\end{tabular}


Table 4 Control design variables for integrated design, with dynamic dissipative controllers.

\begin{tabular}{cccccc}
\hline \hline Var. No. & $\begin{array}{c}\text { Control- } \\
\text { Optimized } \\
\text { Design }\end{array}$ & $\begin{array}{c}\text { Integrated } \\
\text { Design }\end{array}$ & Var. No. & $\begin{array}{c}\text { Control- } \\
\text { Optimized } \\
\text { Design }\end{array}$ & $\begin{array}{c}\text { Integrated } \\
\text { Design }\end{array}$ \\
\hline$A_{c[2,1]}$ & 136.57 & 135.39 & $Q_{[1,1]}$ & 15287.63 & 18122.76 \\
$A_{c[2,2]}$ & 105.25 & 114.73 & $Q_{[2,2]}$ & 30050.42 & 12173.74 \\
$A_{c[1,3]}$ & 136.60 & 301.81 & $Q_{[3,3]}$ & 15125.39 & 10430.10 \\
$A_{c[1,4]}$ & 104.33 & 102.17 & $Q_{[4,4]}$ & 29103.43 & 13600.71 \\
$A_{c[6,5]}$ & 103.80 & 80.42 & $Q_{[5,5]}$ & 19640.50 & 24674.52 \\
$A_{c[6,6]}$ & 107.72 & 125.07 & $Q_{[6,6]}$ & 24331.62 & 19487.68 \\
$A_{c[8,7]}$ & 94.56 & 58.21 & $Q_{[7,7]}$ & 20582.68 & 22964.97 \\
$A_{c[8,8]}$ & 121.26 & 103.52 & $Q_{[8,8]}$ & 22159.79 & 21224.58 \\
$A_{c[10,9]}$ & 136.92 & 95.28 & $Q_{[9,9]}$ & 15148.89 & 22693.96 \\
$A_{c[10,10]}$ & 103.87 & 107.26 & $Q_{[10,10]}$ & 29362.52 & 20381.06 \\
$A_{c[12,11]}$ & 100.11 & 136.11 & $Q_{[11,11]}$ & 20009.49 & 17783.45 \\
$A_{c[12,12]}$ & 126.11 & 133.30 & $Q_{[12,12]}$ & 21511.89 & 15376.09 \\
\hline
\end{tabular}




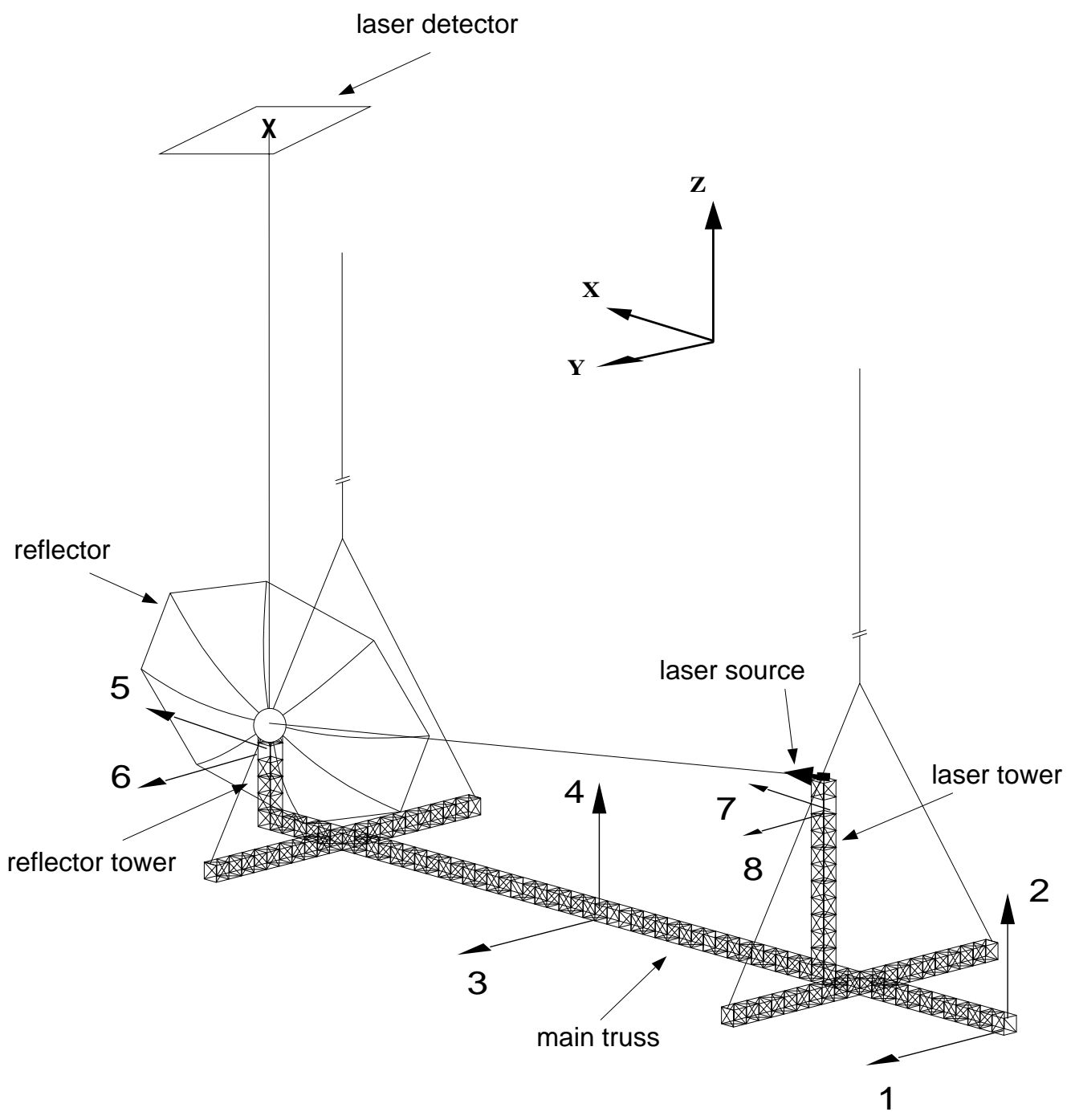

Fig. 1 Schematic of the CEM testbed. 


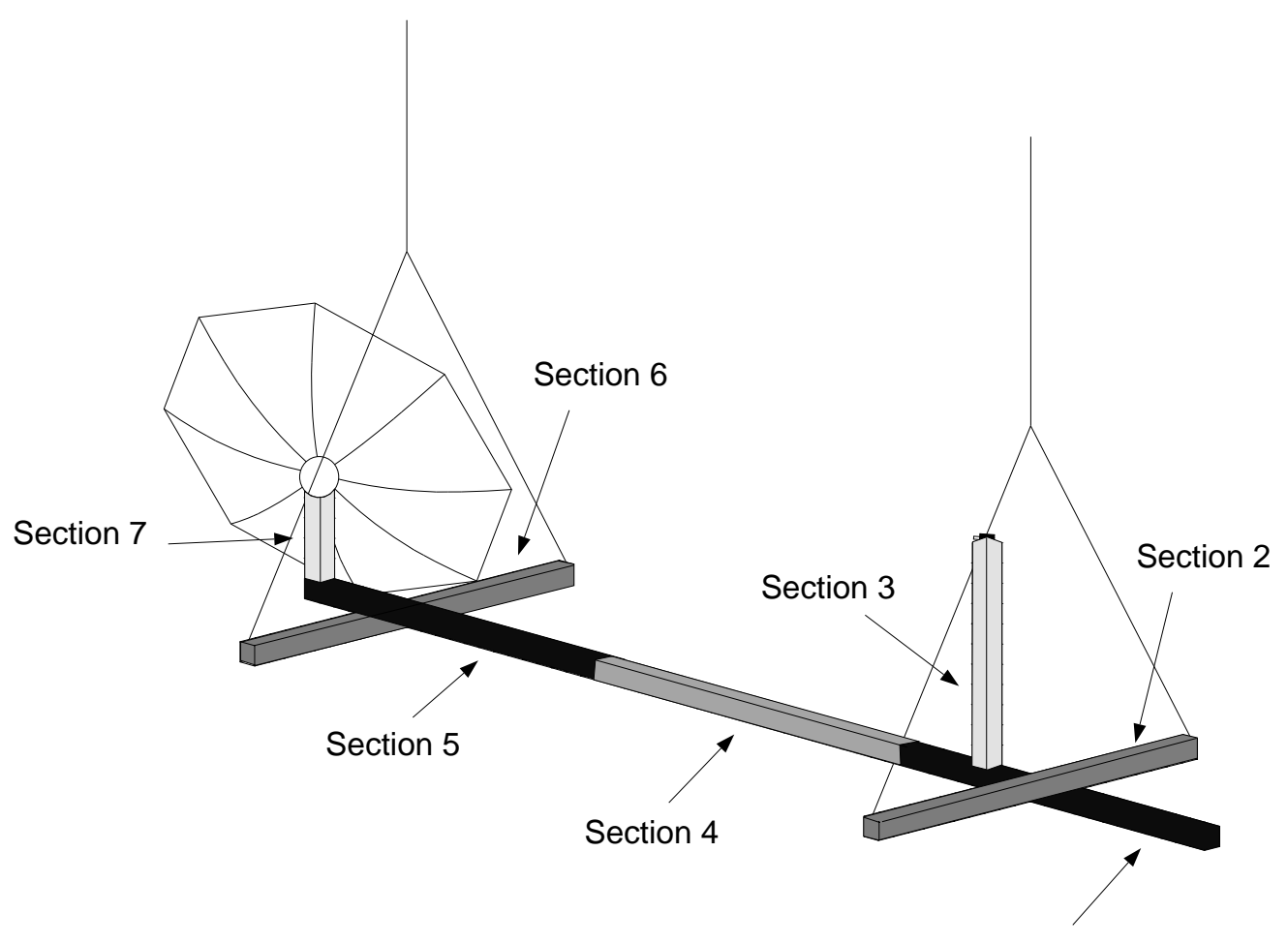

Section 1

Fig. 2 CEM displaying seven sections for integrated redesign. 


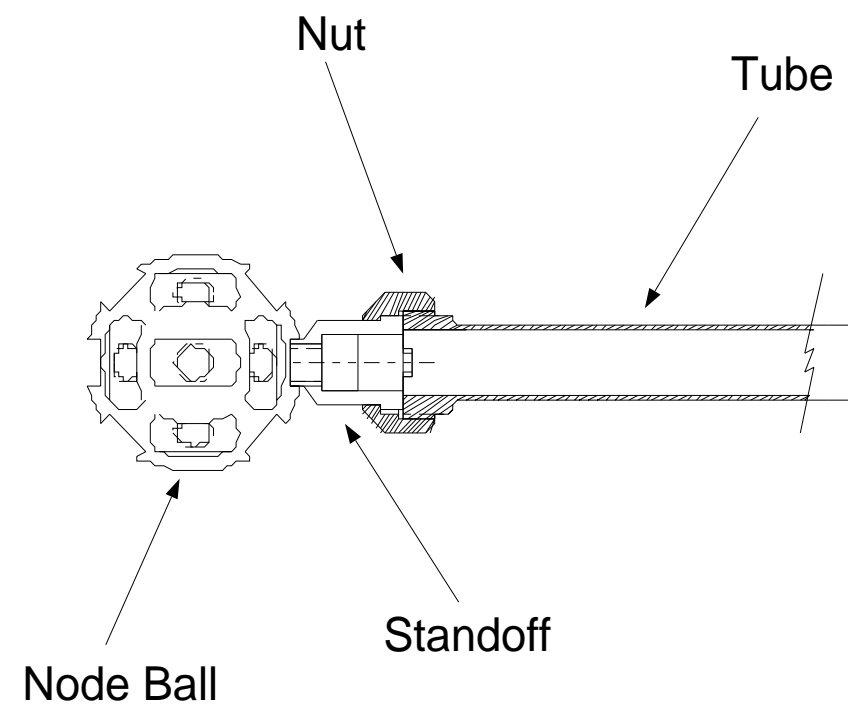

Fig. 3 Strut design for phase-1 CEM. 


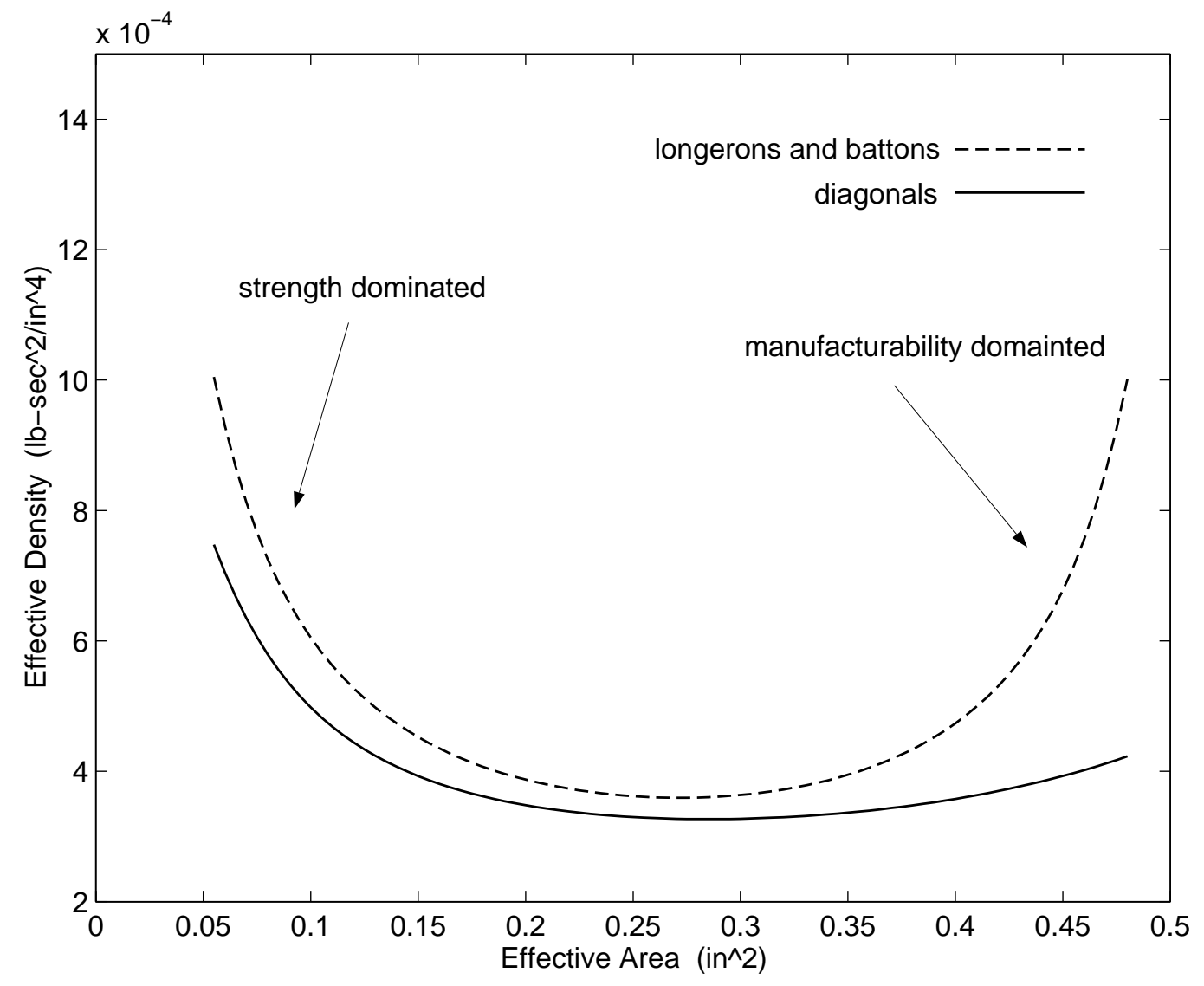

Fig. 4 Strut design curves, longerons and battens (dashed line) and diagonals (solid line). 


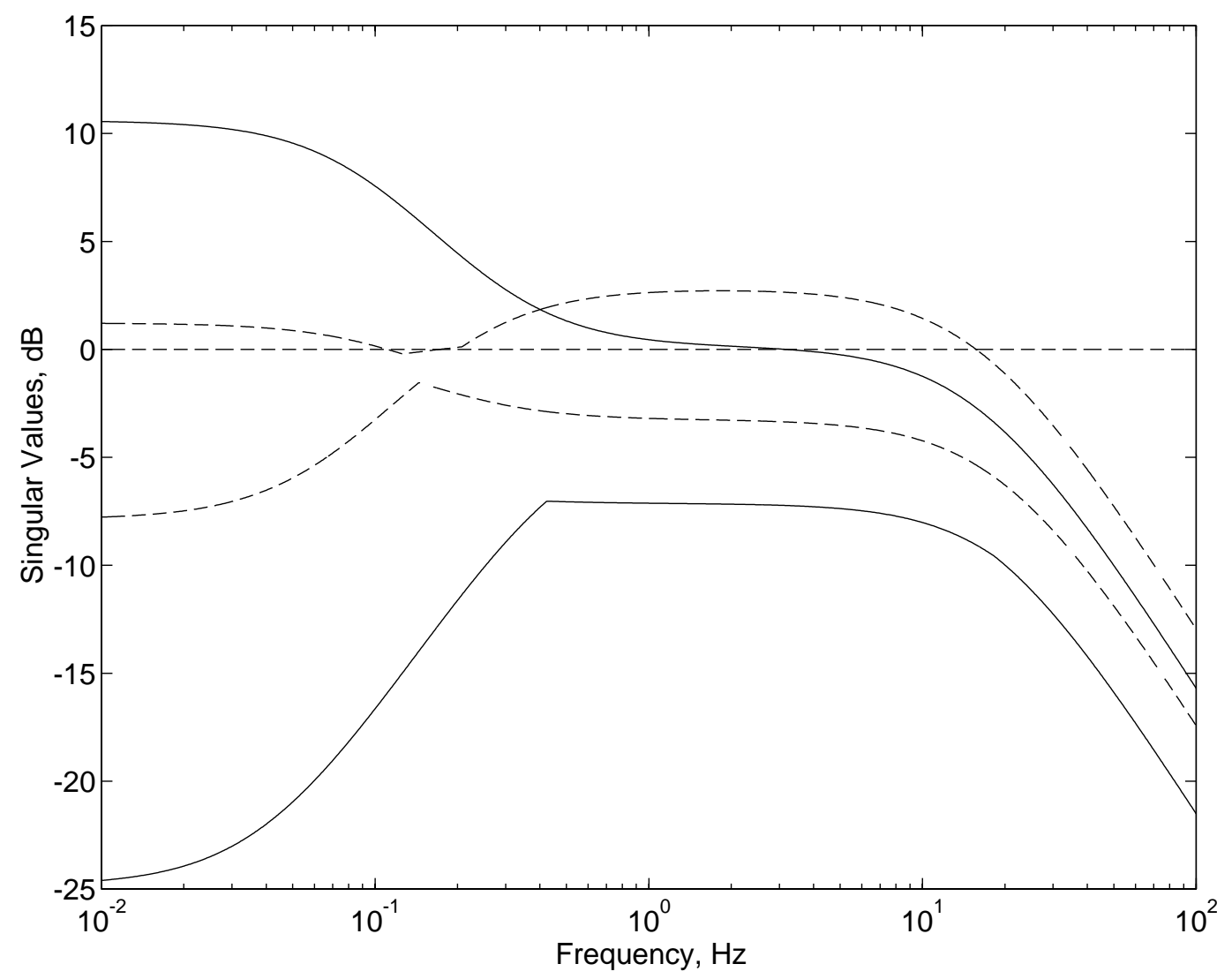

Fig. 5 Maximum and minimum singular value plots of dynamic dissipative controllers for phase-0 CEM (dashed line) and Phase-1 CEM (solid line). 